\title{
Welcher Asthmatyp ist Ihr Patient?
}

\author{
Das Asthma bronchiale ist kein einheitliches Krankheitsbild. Die spezifische Phänotypisierung \\ eröffnet die Möglichkeit für eine gezielte individualisierte Therapie mit Biologika. Doch wann \\ sollte welche der Substanzen zum Einsatz kommen?
}

\begin{abstract}
Oh, wie war es ehedem doch nur mit zwei Asthmatypen so bequem! Doch die Zeiten als man beim Asthma bronchiale nur das allergische vom nicht allergischen unterschied, ohne dass sich daraus besondere differenzialtherapeutische Konsequenzen ergeben hätten, sind vorbei. „Asthma ist nicht Asthma“, so Prof. Christian Taube von der Universität Leiden, Niederlande. Asthma sei ein Syndrom, wobei klinisch ein allergisches, ein nicht allergisches, ein early onset, ein berufsbedingtes und ein schweres allergisches Asthma unterschieden würden. Heute gebe es aber Möglichkeiten für eine weitere spezifische Phänotypisierung jenseits des klinischen Erscheinungsbildes. Dies ist nötig, da bei der klinischen Phänotypisierung große Überschneidungen bestehen und somit eine klare Abgrenzung kaum möglich ist.

Die moderne Differenzierung orientiert sich sowohl an der Art der Entzündungsreaktion (eosinophile, neutrophile und paucigranulozytäre Entzündung) als auch an der Genexpression in der Lunge (Th2-high, Th2-low). „Das Th2-gesteuerte eosinophile Asthma kann aber sowohl allergisch, also IgE-vermittelt, als auch nicht allergisch sein“, so Taube. Die für die Symptomatik entscheidenden Mediatoren sind immer Interleukin 5 und 13, unabhängig davon, ob es sich um allergisches oder nicht allergisches Asthma handelt. Diese neue Typisierung ermöglicht eine am Phänotyp und so pathogenetisch orientierte zielgerichtete Therapie.
\end{abstract}

\section{Problematisches schweres Asthma}

In der täglichen Praxis sollte man zwischen schwierig zu therapierendem und schwerem refraktärem Asthma unterscheiden. Ursachen für ersteres können schlechte Compliance, psychosoziale Faktoren, eine persistierende Exposition gegenüber Umweltfaktoren oder Allergenen und unbehandelte Komorbiditäten sein. „Ein schweres therapierefraktäres Asthma liegt dann vor, wenn die Symptomatik trotz hochdosierter Therapie und Überprüfung der

Compliance sowie korrekter Inhalationstechnik, Ausschluss von Differenzi-

Bronchialverengung bei Asthma das Kardinalsymptom.

\begin{tabular}{|c|c|c|c|c|}
\hline $\begin{array}{l}\text { Behand- } \\
\text { lung }\end{array}$ & Phänotyp & $\begin{array}{l}\text { Lungen- } \\
\text { funktion }\end{array}$ & $\begin{array}{l}\text { Exazer- } \\
\text { bationen }\end{array}$ & $\begin{array}{l}\text { Sympto- } \\
\text { me }\end{array}$ \\
\hline Anti-IL-5 & $\begin{array}{l}\text { Asthma mit eosinophi- } \\
\text { ler Inflammation }\end{array}$ & $\uparrow$ & $\downarrow$ & $\downarrow$ \\
\hline Anti-IL-5 RC & $\begin{array}{l}\text { Asthma mit eosinophi- } \\
\text { ler Inflammation }\end{array}$ & $\uparrow$ & $\downarrow$ & $\downarrow$ \\
\hline Anti-IL-13 & Th1-induziertes Asthma & $\uparrow$ & $\downarrow$ & $\leftrightarrow$ \\
\hline Anti-IL4Rca & Th1-induziertes Asthma & $\uparrow$ & $\downarrow$ & $\downarrow$ \\
\hline Anti-lgE & Allergisches Asthme & $\uparrow$ & $\downarrow$ & $\downarrow$ \\
\hline
\end{tabular}

aldiagnosen, Therapie der Komorbiditäten und Ausschaltung von Triggerfaktoren unkontrolliert bleibt", so Taube. Daher müsse zunächst die Compliance weiter verbessert werden. Auch sollte man daran denken, ob evtl. noch unerkannte Komorbiditäten die Symptomatik erzeugen könnten, die zunächst optimal behandelt werden müssen. Auch sollten Triggerfaktoren ausgeschaltet und die Exposition gegenüber Allergenen und Umweltfaktoren verhindert werden. ,Wichtig ist, die Asthmadiagnose kritisch zu hinterfragen und Differenzialdiagnosen zu diskutieren“ so Taube.

\section{Biologikum statt oraler Kortikoidtherapie}

Die Leitlinie empfiehlt auf der Therapiestufe 4 die Kombination ICS/LABA plus Tiotropium. „Auf Stufe 5 sollte additiv ein Biologikum, nämlich Anti-IgE oder Anti-IL-5, gegeben werden, als Alternative werden orale Kortikosteroide in der niedrigsten effektiven Dosis genannt", so Traube. Ein Biologikum sei besonders im Hinblick auf die Nebeneffekte der Kortikoide aber die bessere Alternative. Die Wahl des Präparates sollte sich am Phänotyp, genauer an Biomarkern, orientieren ( $\bullet$ Tab.1).

Als Antikörper gegen IgE steht seit vielen Jahren Omalizumab zu Verfügung. Sinnvoll ist dieses Therapieprinzip, wenn bei allergischem Asthma als Biomarker IgE im Serum nachzuweisen ist. Ein neues Prinzip gegen schweres eosinophiles Asthma ist die Blockade von IL 5 durch einen humanisierten monoklonalen Antikörper wie Reslizumab bzw. Mepolizumab oder einen Interleukin-5-Rezeptorblocker wie Benralizumab, der sich noch in der Entwicklung befindet. Die Wirksamkeit und Sicherheit von Reslizumab und Mepolizumab wurden in klinischen Studien geprüft. „Mit diesen Substanzen konnten Symptomatik und Exazerbationsrisiko signifikant reduziert werden, auch die Lungenfunktion besserte sich", so Taube. Noch nicht genau definiert sei die Eosinophilenzahl, die die Anti-IL-5-Therapie sinnvoll mache.

Auch für den Phänotyp „,schweres Asthma mit Th2-vermittelter Entzündung" ist eine spezifische Therapie in Sicht, und zwar mit einem Antikörper gegen IL 13 wie Lebrikizumab oder Tralokinumab bzw. einem IL-4-Rezeptor-alfa-Inhibitor wie Dupilumab. Als Biomarker bei diesem Asthma kann Periostin dienen. Für den Alltag bietet sich, so Taube, folgende Differenzialtherapie bei schwerem nicht kontrolliertem Asthma an:

- Anti-IgE: Klinisch relevante Sensibilisierung mit einem relevanten perennialen Allergen.

- Anti-IgE oder Anti-IL-5: Sensibilisierung, Eosinophile im Blut $>300 / \mu \mathrm{l}$, OCS-Behandlung $>150 / \mu \mathrm{l}$.

- Anti-IL-5: Keine relevante Sensibilisierung, Eosinophile im Blut $>300 / \mu$ l, OCS-Behandlung $>150 / \mu 1$.

Dr. med. Peter Stiefelhagen 